\title{
Differential Phase-Shift-Keying Technique-Based Brillouin Echo-Distributed Sensing
}

\author{
Min Won Lee, Birgit Stiller, Jérôme Hauden, Hervé Maillotte, Carole Roch, Luc Thévenaz, and Thibaut Sylvestre
}

\begin{abstract}
In this letter, we experimentally demonstrate Brillouin echoes-based distributed optical fiber sensing with centimeter spatial resolution. It is based on a differential phase-shift-keying technique using a single Mach-Zehnder modulator to generate a pump pulse and a $\pi$-phase-shifted pulse with an easy and accurate adjustment of delay. The results are compared to those obtained in standard Brillouin echo-distributed sensing system with two optical modulators and clearly show a resolution of $5 \mathrm{~cm}$ in a spliced segment between two fibers by applying a $\pi$-phase-shifted pulse of $500 \mathrm{ps}$.
\end{abstract}

Index Terms-Brillouin echo-distributed sensing, Brillouin optical time-domain analysis.

\section{INTRODUCTION}

$\mathbf{O}$ VER the last two decades, Brillouin scattering has widely been studied for distributed sensing applications using optical fibers [1]-[3]. Two main sensing analyses have been developed for this purpose: Brillouin optical correlation-domain analysis (BOCDA) and Brillouin Optical Time-Domain Analysis (BOTDA). BOCDA offers a very good spatial resolution [4] down to $1 \mathrm{~mm}$ [5]. Nevertheless, it is difficult to perform over a long distance using this technique. On the other hand, BOTDA has attracted particular attention for distributed sensing of temperature and/or strain in petrol and civil engineering industries. This analysis offers fast measurement and long-haul distributed sensing by measuring Brillouin frequency shift (BFS) produced by temperature and/or strain in optical fibers [6], [7]. Recently, enhanced BOTDA systems have been reported such as vector [8] and simplex-coded BOTDA systems [9]. Basically, the technique uses a pump-probe counter-propagative configuration with a pulse longer than $10 \mathrm{~ns}$ for pump. However, its spatial resolution is limited to $1 \mathrm{~m}$ due to the acoustic decay time of $10 \mathrm{~ns}$. In order to overcome this limit,

Manuscript received June 24, 2011; revised September 01, 2011; accepted October 06, 2011. Date of publication October 14, 2011; date of current version December 21, 2011. This work was supported by the European INTERREG IVA Program, by the Conseil Régional de Franche-comté, and by the European COST action 299-FIDES.

M. W. Lee, B. Stiller, H. Maillotte, C. Roch, and T. Sylvestre are with the Département d'Optique P. M. Duffieux, Institut FEMTO-ST, Université de Franche-Comté, CNRS UMR 6174, F-25030 Besançon Cedex, France (e-mail: thibaut.sylvestre@univ-fcomte.fr).

J. Hauden is with PHOTLINE Technologies, F-25001 Besançon, France.

L. Thévenaz is with the Institute of Electrical Engineering, Ecole Polytechnique Fédérale de Lausanne, CH-1015, Lausanne, Switzerland.

Color versions of one or more of the figures in this letter are available online at http://ieeexplore.ieee.org.

Digital Object Identifier 10.1109/LPT.2011.2172255 other techniques have been developed such as dark-pulse Brillouin scattering [10], differential pulse-width pair BOTDA [11] and Brillouin echo-distributed sensing (BEDS) [12], [13]. They all have neither physical nor technical limit down to $1-\mathrm{cm}$ resolution sensing. However, in the dark-pulse sensing technique, residual peaks appear in the measurement spectra whereas the other two techniques suppress such peaks. Recently, it has been demonstrated that the BEDS approach offers a double contrast with respect to the other techniques [13]. This technique uses an optical short $\pi$-phase pulse in addition to a long intensity pulse and centimeter resolution sensing can be achieved by the short phase pulse with a very good quality. Despite of the advantage over high spatial resolution, the conventional BEDS systems exploit two modulators: a phase modulator for generating short $\pi$-phase pulses and an intensity modulator for generating long intensity pulses. Besides, measurements can be undertaken using a differential gain approach taking the difference between two successive measurements with and without the $\pi$-phase pulse. Therefore, a good accuracy sensing requires a sophisticated adjustment of the delay between the two pulses.

In this work, we propose a new concept to perform BEDS in which an optical $\pi$-phase pulse is directly generated using a single intensity modulator by differential phase-shift keying (DPSK) technique. The system we propose in this letter is to implement BEDS with an optical scheme as simple as other high spatial resolution techniques, but with a double contrast. A long positive and a short negative electrical pulses are applied to the modulator to generate an optical $\pi$-phase shift and centimeter resolution sensing is achieved in our DPSK-based BEDS system (DPSK-BEDS). Moreover, the pulse delay can easily be adjusted as the pulses are generated in intensity. For this purpose, we investigate a fiber section where two different fibers are spliced with a $5 \mathrm{~cm}$-protection sleeve. The results will be compared with those obtained in a conventional BEDS system. Elongation measurements over a $5-\mathrm{cm}$ fiber section will also be carried out using DPSK-BEDS.

\section{II. $\pi$-Phase Pulse Generation BASEd on DPSK TeChNiQue}

DPSK technique is widely used in telecommunication industry to transmit bit-sequence messages in form of $\pi$-phase shift using an intensity modulator, i.e. Mach-Zehnder interferometer modulator (MZI). In the principle of DPSK, optical phase can be shifted by modulating an MZI with an amplitude of double half-wave voltage $\left(2 V_{\pi}\right)$. When the MZI is driven at its minimum bias point by a positive voltage $0<V<V_{\pi}$ and successively a negative voltage $0>V>-V_{\pi}$, the relative phase-shift between the optical fields at the two voltages is $\pi$, 


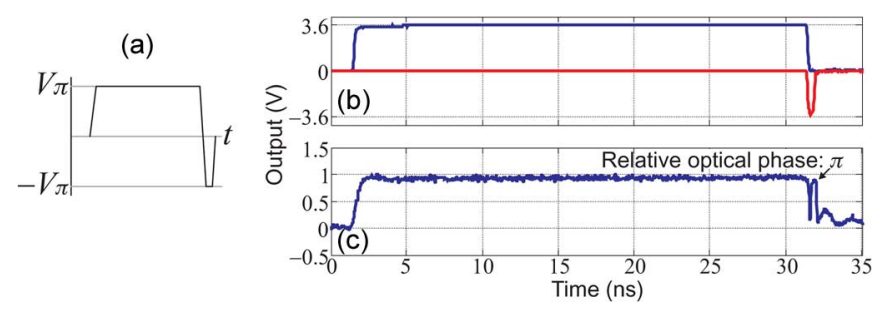

Fig. 1. (a) Schematic drawing of a positive/negative electrical pulse. (b) Positive (blue trace) and negative (red trace) electrical pulses are generated separately and applied to the MZI. The durations of the long and short intensity pulses are $30 \mathrm{~ns}$ and $500 \mathrm{ps}$, respectively. (c) The intensity pulses at the MZI output. The phase is shifted by $\pi$ for $500 \mathrm{ps}$ at the pulse end.

or vice-versa [14]. Therefore, when a negative pulse of $V_{\pi}$ (or a pulse of $-V_{\pi}$ ) is applied to the modulator just after a positive pulse of $V_{\pi}$ at the minimum bias point as illustrated in Fig. 1(a), the output optical intensity remains unchanged whilst the phase of the optical field at the negative pulse part is shifted by $\pi$ with respect to the positive pulse part. In order to generate such an electrical pulse, the positive and negative electrical pulses are generated separately in this work as shown in Fig. 1(b). Their peak-to-peak amplitude is $7.6 \mathrm{~V}$ and applied to the MZI used in the work (Photline MXPE series, $V_{\pi}=6.2 \mathrm{~V}, 20-\mathrm{GHz}$ bandwidth). The pulse durations are set to $30 \mathrm{~ns}$ and $500 \mathrm{ps,}$ respectively. In telecommunication, such pulses are applied to a dual-drive MZI [15]. In our work, these long and short pulses are applied to the direct current (DC) and radio-frequency (RF) inputs of the MZI, respectively. An impedance matching of $50 \Omega$ is made at the DC port and it provides a sufficient bandwidth for the 30-ns pulse. As the pulses are generated in intensity at the output of the MZI, the delay between the pulses is readily adjusted. Fig. 1(c) exhibits two intensity pulses at the modulator output, which are generated by the electrical pulses seen in Fig. 1(b). A 30-ns intensity pulse is followed by a 500-ps intensity pulse with $\pi$-shifted phase. The rise/fall times of the long and short pulses are $700 \mathrm{ps}$ and $200 \mathrm{ps}$, respectively. A short drop between the two pulses is seen due to the fast transition from the positive pulse to the negative pulse. The 500-ps duration of the short pulse defines a spatial resolution of $5 \mathrm{~cm}$ in our DPSK-based BEDS system.

\section{EXPERIMENTAL SETUP}

This dual-phase state intensity pulse is used as the pump wave in the experimental setup shown in Fig. 2 schematically. The setup is based on BOTDA system with an additional electrical pulse which is negative and applied to the MZI of the pump. The continuous wave $(\mathrm{CW})$ emission of a distributed feedback (DFB) laser at $1550 \mathrm{~nm}$ is split into the pump and probe arms by a 50:50 tap coupler. In the pump side, an RF bias-T is used to combine the positive pulse and DC bias and the DC bias of the MZI is adjusted to be at the minimum bias point. The optical pulses in Fig. 1(c) are amplified by an Erbium-doped fiber amplifier (EDFA) with a peak power of $1.3 \mathrm{~W}$. They are then polarization-scrambled by a polarization scrambler to average out polarization-sensitive Brillouin gain and injected into a fiber under test via an optical circulator. In the probe side, the Brillouin frequency-shifted probe is generated by the second MZI with a carrier suppression of $38 \mathrm{~dB}$ at $1550 \mathrm{~nm}$ by adjusting the

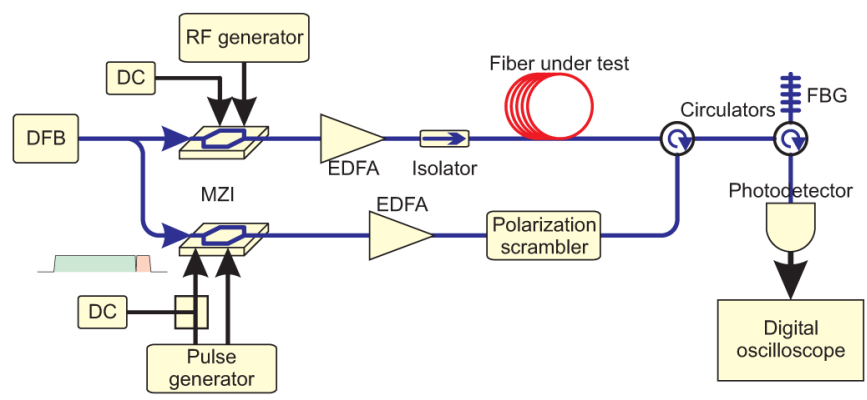

Fig. 2. Experimental setup of DPSK-BEDS system using a single Mach-Zehnder interferometer (MZI) modulator for the pump. DFB: Distributed-feedback laser. EDFA: Erbium-doped fiber amplifier. FBG: Fiber Bragg grating.

DC bias. The probe power is set to $1 \mathrm{~mW}$ by another EDFA. The probe is then injected into the fiber through an isolator. At the fiber output, a fiber Bragg grating (FBG) enables to select the Brillouin gain obtained by the pump at the BFS. The gain is retrieved by a photodetector and then recorded as time traces by a digital oscilloscope. By sweeping the frequency of the optical probe, the gain along the fiber is mapped as a function of frequency. In this way, all BFS variations along the fiber caused by temperature or strain can be easily detected.

\section{EXPERIMENTAL RESULTS}

In order to demonstrate the performance of the concept developed in this work, a 2-m single-mode fiber (SMF) is spliced to a 1-m fiber with high numerical aperture (HNA). The splice point is protected by a heat-shrinking sleeve of $5 \mathrm{~cm}$. For comparison, distributed measurements of the splice segment have been done in two BEDS systems: conventional BEDS system using a phase and an intensity modulators for pump and DPSK-BEDS system using a single intensity modulator. Fig. 3(a) shows the distributed measurement obtained in the conventional BEDS system via a $\pi$-phase shifted pulse with a pulse width of $500 \mathrm{ps}$. It clearly reveals the splice segment of $5 \mathrm{~cm}$ between the fibers at a frequency shift of $10.55 \mathrm{GHz}$. The BFS of the HNA and SMF are $10.67 \mathrm{GHz}$ and $10.85 \mathrm{GHz}$, respectively. Fig. 3(b) displays the mapping using DPSK-BEDS developed in the work. It also manifests clearly the $5-\mathrm{cm}$ splice segment and the $10.55-\mathrm{GHz}$ frequency shift. Therefore, it is evident that our system manifests performances as good as the standard BEDS system. However, the contrast of the measurement in BEDS is slightly better than that in DPSK-BEDS. This is due to the peak-to-peak amplitude of $7.6 \mathrm{~V}$ which is only $1.23 V_{\pi}$ in DPSK-BEDS where $2 V_{\pi}$ is needed for the best performance. On the other hand, in BEDS a pulse amplitude of $5.1 \mathrm{~V}$ is applied to the phase modulator of which the $V_{\pi}$ is $5.9 \mathrm{~V}$. In this case, only $V_{\pi}$ is required for the best operation and the amplitude meets almost this requirement $\left(0.87 V_{\pi}\right)$. Nevertheless, Fig. 3(b) shows a good margin for improvement thanks to the good signal-to-noise ratio.

We have also tested our DPSK-BEDS system with centimeter resolution for strain monitoring. For that purpose, we have set up an elongation platform and applied three different elongations over a fiber section of $5 \mathrm{~cm}$ in the beginning of a $260-\mathrm{m}$ long SMF. Fig. 4 exhibits the elongation measurements with $5-\mathrm{cm}$ spatial and 1-MHz spectral resolutions. The elongations applied 

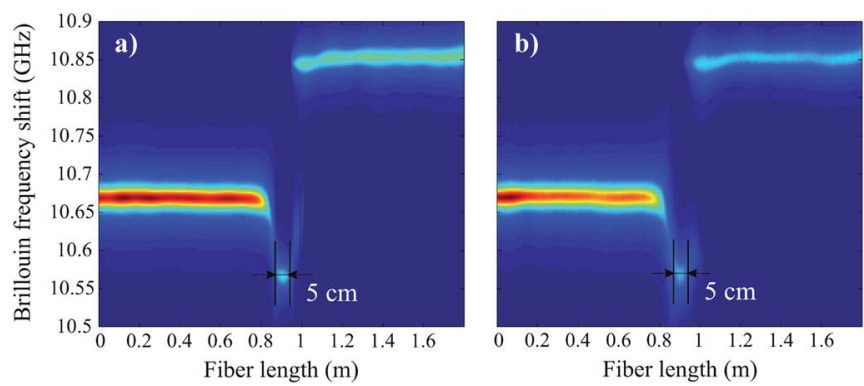

Fig. 3. Distributed measurements of HNA/SMF fibers showing a splice segment of $5 \mathrm{~cm}$ (a) in standard BEDS system using a phase modulator and (b) in our BEDS system based on DPSK technique.

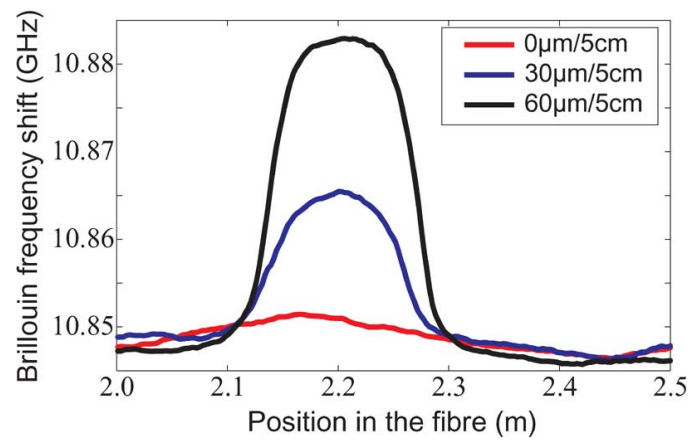

Fig. 4. Brillouin frequency shift versus the fiber length for three different elongations applied to a 5-cm fiber section.

to the section are successively $0 \mu \mathrm{m}, 30 \mu \mathrm{m}$ and $60 \mu \mathrm{m}$. As the elongation length increases, the BFS increases from 10.847 $\mathrm{GHz}$ to $10.865 \mathrm{GHz}$ and $10.883 \mathrm{GHz}$. However, the figure reveals the elongation over more than $5 \mathrm{~cm}$. We speculate that the pure fiber may slightly slip inside the fiber coating as a result of strain. The BFS is displaced by $18 \mathrm{MHz}$ at $600 \mu \varepsilon$ and 36 $\mathrm{MHz}$ at $1200 \mu \varepsilon$. Thus, the strain coefficient is obtained as 3 MHz per $100 \mu \varepsilon$ with a strain accuracy of $33 \mu \varepsilon$. This accuracy can be further improved by using more frequency samples and higher pump and probe power. Since it is based on BEDS technique, our setup can potentially reach spatial resolution down to $1 \mathrm{~cm}$ if a faster pulse generator is used.

\section{CONCLUSION}

We have successfully demonstrated a new concept for Brillouin echo-distributed sensing with centimeter resolution based on differential phase-shift keying technique. A $\pi$-phase pulse with a pulse duration of $500 \mathrm{ps}$ and an intensity pulse with $30 \mathrm{~ns}$ are generated for the pump using a single Mach-Zehnder modulator in our system. Our results clearly reveal the $5-\mathrm{cm}$ splice segment between two fibers and its Brillouin frequency shift with a spatial resolution of $5 \mathrm{~cm}$. The elongation test over a fiber section of $5 \mathrm{~cm}$ has also been performed with good results. Our new setup enables distributed measurements with centimeter resolution and a double contrast by simply adding a negative pulse into the pump in a Brillouin optical time-domain analysis system. Therefore it simplifies conventional Brillouin echo-distributed sensing systems by using a single modulator for pump rather than two modulators, and reduces the optical loss of the pump due to the modulators. Our setup also allows an easy adjustment of the delay between two pulses as they are generated in intensity. As such, our system may offer a great potential to fiber optic sensors with centimeter resolution for structural health and geotechnical monitoring.

\section{REFERENCES}

[1] T. Kurashima, T. Horiguchi, and M. Tateda, "Distributed-temperature sensing using stimulated Brillouin scattering in optical silica fibers," Opt. Lett., vol. 15, pp. 1038-1040, 1990.

[2] X. Bao, D. J. Webb, and D. A. Jackson, "Combined distributed temperature and strain sensor based on Brillouin loss in an optical fiber," Opt. Lett., vol. 19, pp. 141-143, 1994.

[3] K. Y. Song and H. J. Yoon, "High-resolution Brillouin optical time domain analysis based on Brillouin dynamic grating," Opt. Lett., vol. 35 , pp. 52-54, 2010

[4] K. Hotate and M. Tanaka, "Distributed fiber Brillouin strain sensing with 1-cm spatial resolution by correlation-based continuous-wave technique," IEEE Photon. Technol. Lett., vol. 14, no. 2, pp. 179-181, Feb. 2002.

[5] K. Y. Song, Z. He, and K. Hotate, "Distributed strain measurement with millimeter-order spatial resolution based on Brillouin optical correlation domain analysis," Opt. Lett., vol. 31, pp. 2526-2528, 2006.

[6] F. Rodríguez-Barrios, S. Martín-López, A. Carrasco-Sanz, P. Corredera, J. D. Ania-Casta nón, L. Thévenaz, and M. González-Herráez, "Distributed Brillouin fiber sensor assisted by first-order Raman amplification," J. Lightw. Technol., vol. 28, no. 15, pp. 2162-2172, Aug. 1, 2010.

[7] M. A. Soto, G. Bolognini, and F. Di Pasquale, "Long-range simplexcoded BOTDA sensor over $120 \mathrm{~km}$ distance employing optical preamplification," Opt. Lett., vol. 36, pp. 232-234, 2011.

[8] M. Dossou, D. Bacquet, and P. Szriftgiser, "Vector Brillouin optical time-domain analyzer for high-order acoustic modes," Opt. Lett., vol. 35, pp. 3850-3852, 2010.

[9] M. A. Soto, G. Bolognini, F. D. Pasquale, and L. Thévenaz, "Simplexcoded BOTDA fiber sensor with 1-m spatial resolution over a 50-km range," Opt. Lett., vol. 35, pp. 259-261, 2010.

[10] A. W. Brown, B. G. Colpitts, and K. Brown, "Dark-pulse Brillouin optical time-domain sensor with 20-mm spatial resolution," J. Lightw. Technol., vol. 25, no. 1, pp. 381-386, Jan. 2007.

[11] W. Li, X. Bao, Y. Li, and L. Chen, "Differential pulse-width pair BOTDA for high spatial resolution sensing," Opt. Express, vol. 16, pp. 21616-21625, 2008

[12] L. Thévenaz, "Brillouin distributed time-domain sensing in optical fibers: State of the art and perspectives," Frontiers Optoelectron. China, vol. 3, pp. 13-21, 2010.

[13] S. M. Foaleng, M. Tur, J. Beugnot, and L. Thévenaz, "High spatial and spectral resolution long-range sensing using Brillouin echoes," $J$. Lightw. Technol., vol. 28, no. 20, pp. 2993-3003, Oct. 15, 2010.

[14] G. Charlet, "Progress in optical modulation formats for high-bit rate WDM transmissions," IEEE J. Quantum Electron., vol. 12, no. 4, pp. 469-483, Jul./Aug. 2006.

[15] Y. J. Wen, A. Nirmalathas, and D.-S. Lee, "RZ/CSRZ-DPSK and chirped NRZ signal generation using a single-stage dual-electrode Mach-Zehnder modulator," IEEE Photon. Technol. Lett., vol. 16, no. 11, pp. 2466-2468, Nov. 2004 